\title{
O Sistema de Transformações Cromáticas no Interlúdio de Artémis de Alberto Nepomuceno
}

\author{
The Chromatic Transformations System in the Interlude from Artémis, by \\ Alberto Nepomuceno
}

\author{
Rita de Cássia Taddei \\ Universidade de São Paulo
}

\begin{abstract}
Resumo: Este artigo apresenta alguns conceitos das teorias de Riemann e de Kopp que são aplicados à análise estrutural do Interlúdio de Artémis, episódio lírico composto por Alberto Nepomuceno em 1898. A investigação analítica esclarece que a ferramenta proposta por Kopp-sistema de transformações cromáticas-ajusta-se significativamente bem ao texto musical analisado. A análise aponta que o Interlúdio de Artémis é estruturado com fórmulas cadenciais ortodoxas, porém com emprego de acordes alterados que resultam no uso de tonalidade expandida. Além de aplicar essa ferramenta analítica ainda pouco utilizada nos estudos da música brasileira, este trabalho demonstra que ela ajuda a compreender aspectos técnicos importantes do repertório do final do século XIX e começo do século XX. Sua aplicação é particularmente apropriada em obras de compositores que estiveram à frente de seu tempo no que se refere a pesquisar e aplicar técnicas composicionais inovadoras como as transformações cromáticas, que representavam a vanguarda de seu tempo.
\end{abstract}

Palavras-chave: Nepomuceno; Transformações cromáticas; Análise estrutural

\begin{abstract}
This paper presents some theoretical concepts of Riemann and Kopp applied to the structural analysis of the Interlude of Artémis, a lyrical operatic episode composed by Alberto Nepomuceno in 1898. The analytical investigation clarifies that the analytical tool proposed by Kopp - the system of chromatic transformations - fits remarkably well to the studied musical text. The analysis points out that the Interlude of Artémisis was structured with orthodox cadential formulas, although featuring altered chords, which result in the use of expanded tonality. Besides employing this analytical tool, still seldom employed in the study of Brazilian music, this research demonstrates how it helps to understand important technical aspects of the repertoire of the late XIX Century and early XX Century. Its application is particularly elucidative in works of composers who were ahead of their time in respect to the research and use of innovative compositional techniques as chromatic transformations, which belong to the vanguard of their time.
\end{abstract}

Keywords: Nepomuceno; Chromatic Transformations; Structural Analysis 


\section{Introdução}

Este artigo alicerça-se na ideia de congregar dois teóricos e um compositor que estiveram à frente de seu tempo no que tange à pesquisa e aplicação de técnicas composicionais de vanguarda: Riemann, Kopp e Nepomuceno. É significativo como a teoria analítica elaborada por Kopp, oriunda de uma substancial pesquisa nos escritos de Riemann, ajusta-se coerentemente ao texto musical de Nepomuceno.

Para cumprir esse propósito, apresentaremos, inicialmente, alguns pontos das teorias de Riemann e Kopp, para posteriormente aplicá-las na análise do Interlúdio do episódio lírico Artémis, de Nepomuceno.

A partir dos escritos de Riemann, nossa explanação parte dos conceitos de função e cadência que, ao longo da história da teoria musical, foram tomados com vários significados. Esclarecemos, desta forma, como esses dois termos são considerados dentro da metodologia analítica deste trabalho.

Explicados esses dois termos, com base nos apontamentos de Riemann, avançamos, de forma concisa, para a teoria de Kopp, denominada sistema de transformações cromáticas e planejada para ser empregada em textos musicais com uso de cromatismo.

A parte final deste artigo consiste na análise propriamente dita do Interlúdio de Artémis, validando a asserção de que a estrutura do texto musical e a ferramenta de análise utilizada são congruentes.

\section{Os termos função e cadência segundo Riemann}

Nomeado funktion por Riemann no final do século XIX, o termo se referia à forma para explicar como todos os acordes, diatônicos ou cromáticos, têm um significado dentro da tonalidade que os converge para uma das três funções designadas pelas tríades principais: tônica, subdominante ou dominante. Função, portanto, indicava a potencialidade de um dado acorde em realizar uma progressão a um acorde pré-definido, como é o caso da relação dominante-tônica, por exemplo.

Para o teórico, função harmônica não designava um acorde em si, mas uma interpretação deste acorde que explica como ele se relaciona em seu contexto métrico tonal, esclarecendo que existe um acorde central-um eixo-que conecta acordes utilizados em determinada harmonização (seja ela realizada em modo 
maior como em modo menor), o que confirma uma lei natural que define a função desses acordes. Os principais acordes interligados a esse eixo são a dominante e a subdominante ( $5^{\underline{a}}$ superior e $5^{\underline{a}}$ inferior, respectivamente) ${ }^{1}$

Riemann afirmava que transformações - alterações cromáticas - em um acorde não alteravam sua função.

Especificamente neste estudo, o termo função denota um sentido significativo em relação a um centro tonal, por incluir acordes alterados cromaticamente que têm relação direta com acordes de conteúdo diatônico ou resolvem em acordes de conteúdo diatônico.

Dentro desta perspectiva, a cadência constitui um contexto no qual função e métrica conjugam-se como forças mutuamente determinantes. Cadência constitui, portanto, um encadeamento de acordes (alterados cromaticamente ou não) que convergem para um centro tonal e cujas funções são determinadas pelo seu papel dentro desta sequência.

\section{A teoria de Kopp}

A teoria da transformação tem sistemas projetados para explicar e aplicar em repertório altamente cromático da segunda metade do século XIX e início do século XX. Somada à teoria de notas comuns, também atribuída à Kopp, esta ferramenta analítica aponta fortemente para reconhecimento e inclusão das relações cromáticas de notas comuns em um modelo musical abrangente de relações harmônicas.

No que se refere à notação, no sistema cromático as transformações são designadas em itálico, enquanto a função não. O senso de tonalidade é sempre mantido e o uso das notações transformacional e funcional simultaneamente objetiva descrever sentido harmônico e movimento.

Conforme apontado na Tabela 1 , as notações empregadas nas transformações embasam-se em relações intervalares de fundamentais, terças ou quintas entre os acordes, os quais são sempre considerados em posição fundamental, com ou sem mudança de modo ou alterações cromáticas.

\footnotetext{
${ }^{1}$ Riemann, Hugo. Armonia e Modulación. Barcelona: Labor, 1930. p. 36.
} 
MUSICA THEORICA Revista da Associação Brasileira de Teoria e Análise Musical 2020,

v. 5, n. 1, p. 278-288 - Journal of the Brazilian Society for Music

Theory and Analysis @ TeMA 2020 - ISSN 2525-5541

Transformações Cromáticas

\begin{tabular}{|c|c|c|c|}
\hline $\begin{array}{c}\text { Intervalo entre as } \\
\text { fundamentais }\end{array}$ & Mesmo modo & Mudança de modo & Observações \\
\hline $\begin{array}{l}\text { Fundamental } \\
\text { (uníssono) }\end{array}$ & $I$ & $P$ & $\begin{array}{l}\text { IDENT (idêntico) preserva o modo do } \\
\text { acorde, e PAR (paralelo) implica na } \\
\text { alteração da terça, convertendo a } \\
\text { tríade maior em menor ou vice versa. }\end{array}$ \\
\hline Terça & $M / m$ & $R / r$ & $\begin{array}{l}M \quad \text { e } m: \text { correspondem, } \\
\text { respectivamente, aos intervalos de } \underline{3 \mathrm{M}} \\
\text { e } \underline{3 \mathrm{~m}} \text {, no sentido descendente. Caso } \\
\text { ocorram no sentido ascendente, } \\
\text { acrescenta-se aos mesmos "-1". } \\
R \text { e } r \text { : correspondem, respectivamente, } \\
\text { aos intervalos de } \underline{3 \mathrm{M}} \text { e } \underline{3 \mathrm{~m}} \text {, nos } \\
\text { sentidos ascendente ou descendente. }\end{array}$ \\
\hline Quinta & $D$ & $F$ & $\begin{array}{l}D \text { e } F \text { : correspondem ao intervalo } \\
\text { descendente de } 5 \text { I (com a diferença de } \\
\text { que no primeiro o modo é mantido e } \\
\text { no segundo, não). Para indicar sentido } \\
\text { ascendente, acrescenta-se "-1". }\end{array}$ \\
\hline
\end{tabular}

Tabela 1: Kopp, Transformações Cromáticas Principais

Para uma melhor compreensão quanto aos numerais que representam movimentações ascendentes ou descendentes, propomos uma representação com eixos cartesianos, como vemos na Figura 1 (o exemplo está considerando a tonalidade de Lá Maior e ilustra relações intervalares de quinta em acordes de mesmo modo-D).

Funções convergindo ascendentemente ou descendentemente em direção ao zero (LÁ)

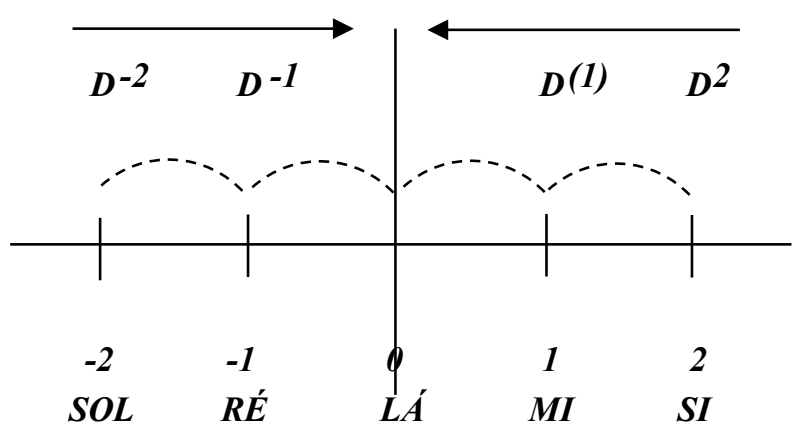

Figura 1: Representação de movimentação ascendente e descendente no sistema de Kopp

A relação intervalar de trítono representa a mais distante relação entre acordes. 
O sistema de Kopp engloba a soma de duas transformações: Slide, movimentação de fundamentais por um intervalo de semitom-unindo tonalidades distantes com mudança de modo-adicionada à transformação Fifth, intervalo de quinta entre as fundamentais dos acordes com mudança de modo. A indicação transformacional entre o acorde menor sobre o segundo grau (ii) em tonalidade maior e o acorde de Napolitana (N) é Slide, ou S. Assim, enquanto a fórmula para ii-V é $\boldsymbol{F}$ (fifth), a progressão N-V é $S F$ (slide fifth), expressão que informa o sentido correto da progressão (Fig. 2).

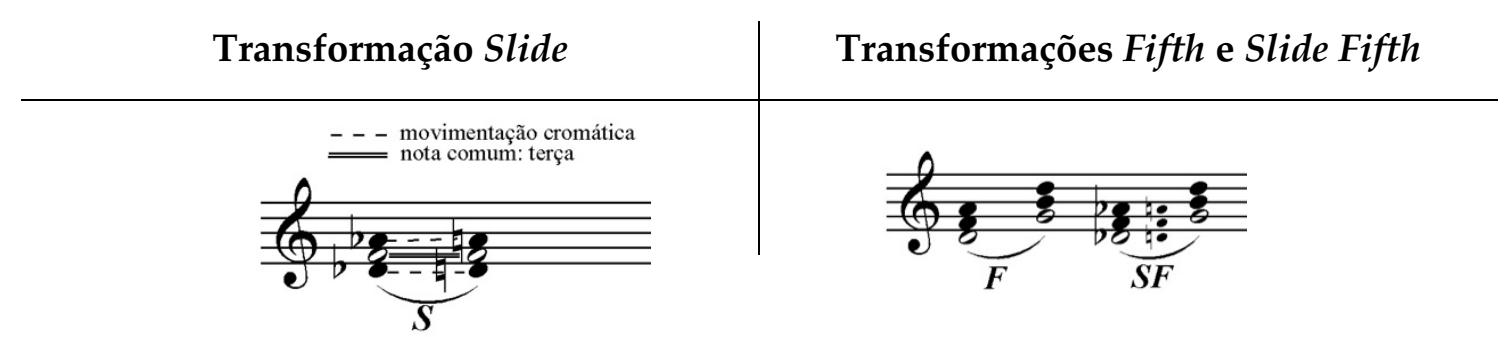

Figura 2: Kopp, Transformações S, F e SF

Kopp complementa o quadro de relações transformacionais acrescentando as compostas, que se estruturam a partir da conexão de duas transformações. Na Figura 3 apontamos as transformações compostas (exemplos escritos na tonalidade de Dó Maior).

Transformações Compostas

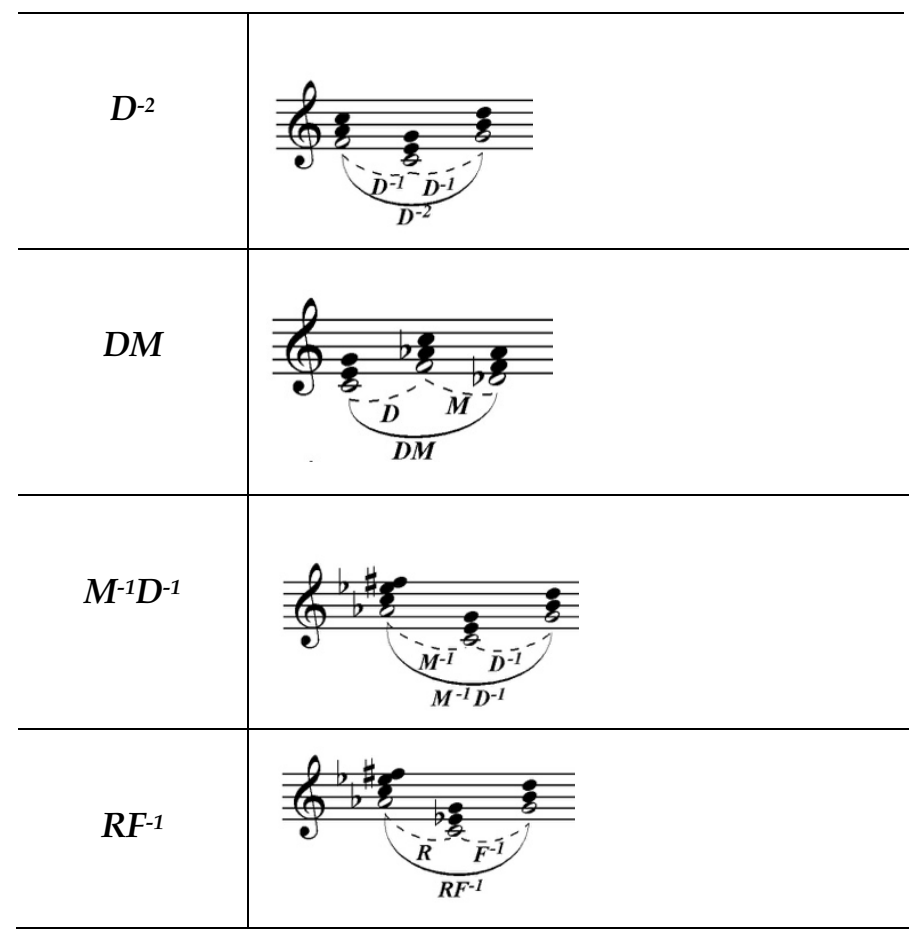

Figura 3: Kopp, Transformações Compostas 


\section{Análise do Interlúdio de Artémis, de Nepomuceno}

O Interlúdio, quarta seção da ópera Artémis, ocorre entre os compassos 626 e 723 e divide as Cenas I/II e III/IV.

Apesar do amplo emprego de acordes alterados, é evidente a simetria ocorrente na construção harmônica desta seção: uma sequência de terças maiores e menores (em movimentação descendente) em torno do centro lá, que é afirmado ao final da seção por meio de um intervalo de trítono.

Esta análise apresenta-se organizada em três fragmentos, subdivididos com base na textura. Inicialmente, expõe-se a análise de cada fragmento. Posteriormente, aponta-se o resumo da estrutura harmônica de toda a seção, ratificando o emprego da ferramenta proposta por Kopp para o processo de análise. Além disso, o resumo estrutural da análise é mostrado no Diagrama de Riemann -Tonnetz-legitimando a conexão existente entre as teorias dos dois teóricos. Conforme já colocado, o Sistema de Transformações Cromáticas é alicerçado na teoria de Riemann.

Quanto ao Interlúdio de Artémis, o primeiro fragmento (c. 626-650) traz a indicação de andamento Lentamente. No que se refere à estrutura harmônica, o trecho, que se inicia com um acorde de Lá Maior, movimenta-se em terças descendentes para Fá\# menor e Ré Maior, chegando, portanto, à subdominante, função na qual permanece por todo o fragmento. Ainda que ao final da seção o acorde de Ré Maior caminhe para Si menor, consideramos, para ambos, a mesma função (subdominante). No Exemplo 1, que mostra a análise do primeiro fragmento, podemos observar a ocorrência de acordes alterados cromaticamente, que indicam o emprego de tonalidade expandida pelo compositor.

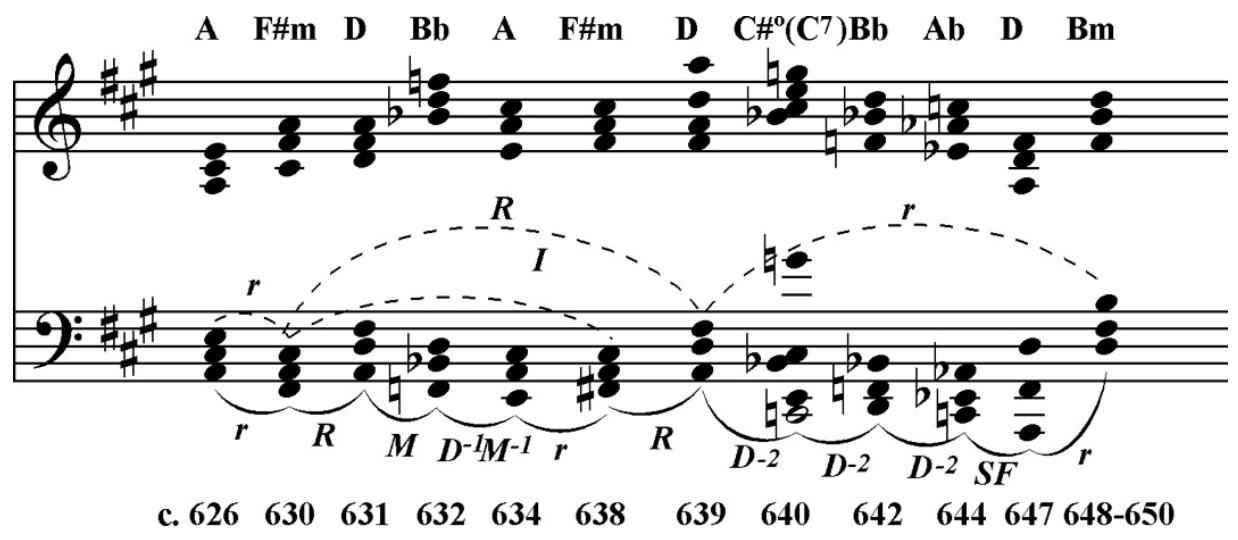

Exemplo 1: Nepomuceno, Artémis - Interlúdio, c. 626-650; análise da estrutura harmônica segundo o sistema de transformações cromáticas 
O segundo segmento do Interlúdio (c. 651-678), vem com duas indicações de andamento: Allegro (c. 651-655) e Moderato (c. 656-678). A estrutura harmônica permanece em Sol Maior, que é prolongado por meio de uma movimentação para Láb Maior. O Exemplo 2 traz a análise do segundo fragmento. Da mesma forma como no exemplo anterior, ocorrem acordes alterados.

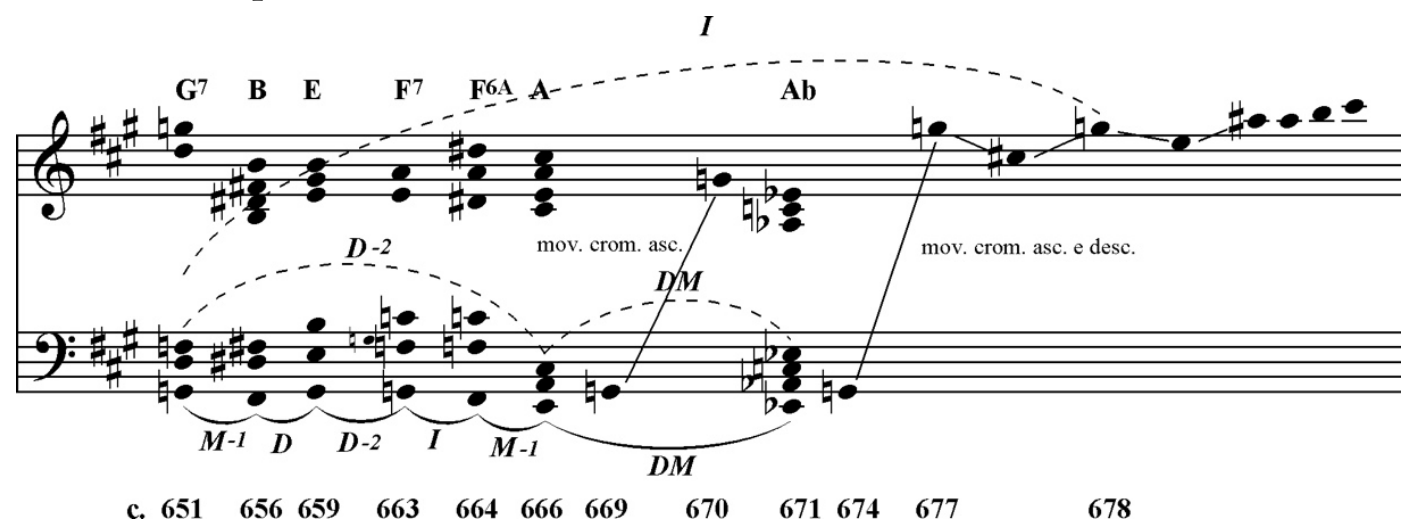

Exemplo 2: Nepomuceno, Artémis - Interlúdio, c. 651-678; análise da estrutura harmônica segundo o sistema de transformações cromáticas

O terceiro fragmento (c. 679-723) aponta os andamentos Dolorosamente (c. 696-706) e Grave come prima (c. 707-723). Neste trecho, como vemos no Exemplo 3, verificamos o retorno ao centro tonal Lá Maior, que é afirmado por meio de uma movimentação de $4^{\mathrm{a}}$ aumentada.

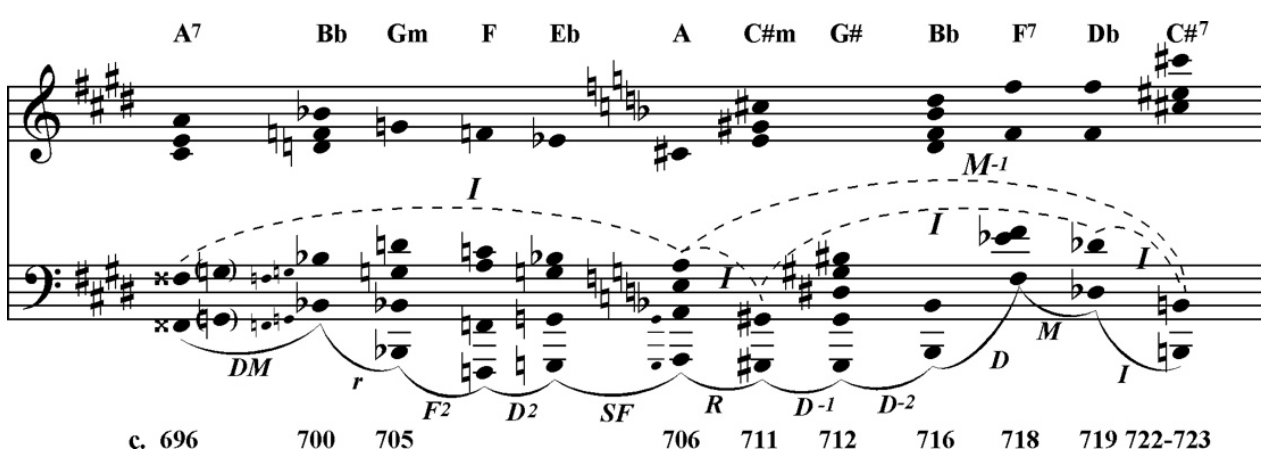

Exemplo 3: Nepomuceno, Artémis - Interlúdio, c. 696-723; análise da estrutura harmônica segundo o sistema de transformações cromáticas

Com base nas análises dos três fragmentos, organizamos o resumo da estrutura harmônica do Interlúdio, que mostra a permanência no centro tonal Lá Maior, com prolongamento de harmonia de subdominante, Ré Maior e retorno a Lá Maior, por meio do acorde de Mi, Maior, portanto sua antípoda. 
MUSICA THEORICA Revista da Associação Brasileira de Teoria e Análise Musical 2020,

v. 5, n. 1, p. 278-288 - Journal of the Brazilian Society for Music

Nota-se, também, que a ponte para a chegada a Mi, Maior se faz pelo acorde de Lá, Maior, acorde este que intermediou o prolongamento de Sol Maior no segundo fragmento desta análise. Este caminho harmônico, Lá Maior-Ré Maior-(Lá, Maior)-Mib Maior-Lá Maior, estrutura uma formação cadencial sobre a qual está construída esta seção de Artémis. No Exemplo 4 mostramos o resumo da estrutura harmônica do Interlúdio.

Exemplo 4:

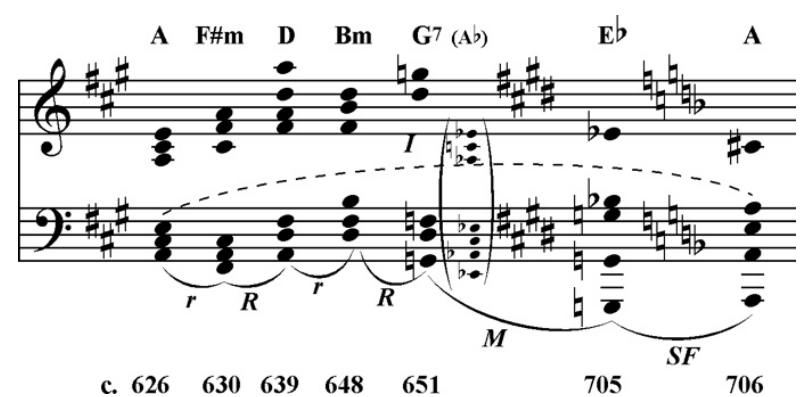

Artémis - Interlúdio, c. 626-706; resumo da estrutura harmônica segundo o sistema de transformações cromáticas

Verificando os principais acordes apresentados no resumo (Exemplo 8), podemos sintetizar a estrutura do Interlúdio em uma formação cadencial, cujo acorde com função de dominante é a antípoda do centro tonal. Salientamos aqui a definição de cadência segundo Riemann, na qual função e métrica harmônica associam-se com potencialidades igualmente determinantes. Apontamos no Exemplo 5 esta cadência com a indicação das transformações cromáticas.

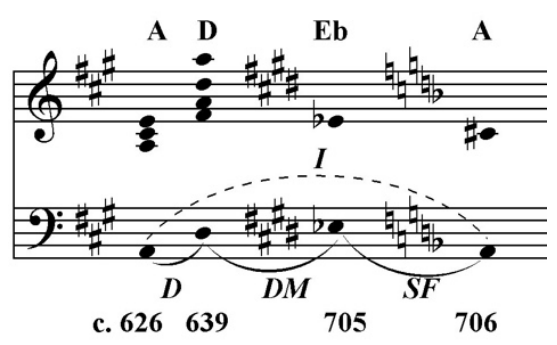

Exemplo 5: Nepomuceno, Artémis - Interlúdio, c. 626-723; síntese cadencial com indicação das transformações cromáticas

No Exemplo 6 indicamos somente as funções, compreendendo toda a seção como convertida a uma cadência. Nesta estrutura a cadência é I-IVV(alterado)-I, o que ratifica a aplicação da teoria de Kopp para este tipo de repertório, que contém amplo emprego de acordes alterados. 


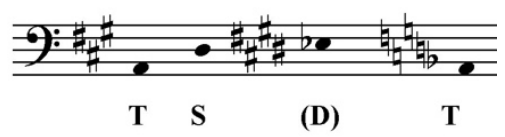

Exemplo 6: Nepomuceno, Artémis - Interlúdio, c. 626-723; síntese cadencial com indicação de funções

Para fechar o processo analítico, organizamos esta estrutura no Tonnetz expandido elaborado por Riemann, de forma a obtermos o diagrama mostrado na Figura 4.

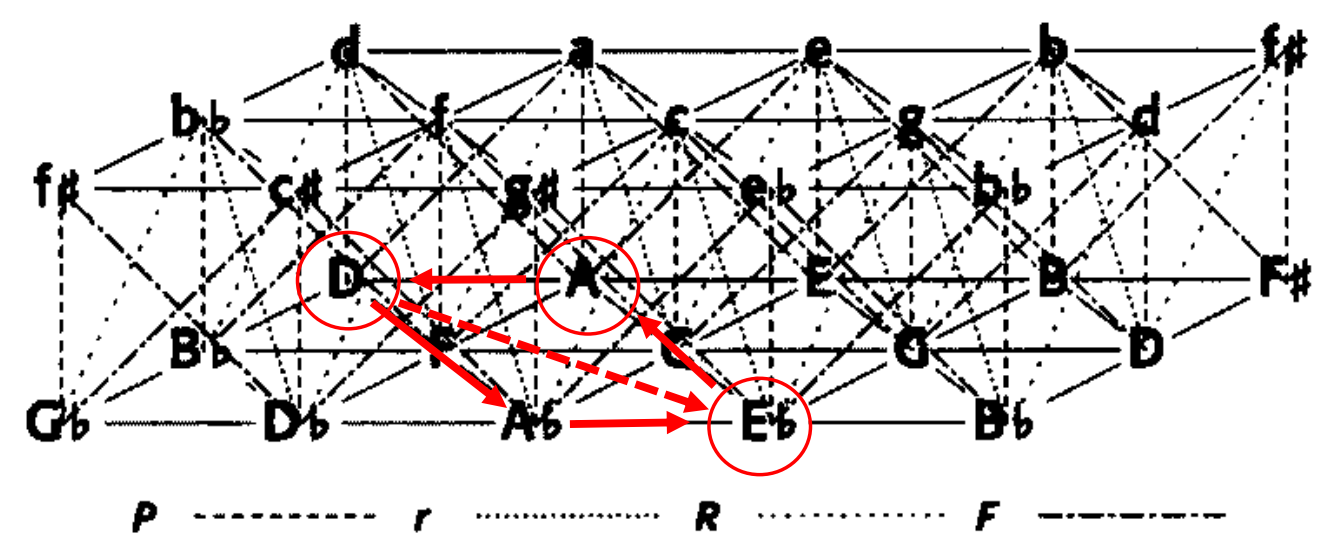

Figura 4: Nepomuceno, Artémis - Interlúdio, c. 626-723; síntese cadencial dentro do Tonnetz expandido de Riemann

Finalizamos a análise organizando a síntese cadencial do Interlúdio no diagrama de conexões proposto por Kopp, no qual notamos a afirmação do centro tonal Lá Maior, a ocorrência da harmonia de subdominante, Ré Maior, a movimentação de Ré Maior para Mib Maior por meio da passagem pelo acorde de Láb Maior e o retorno a Lá Maior por um salto de trítono (Fig. 5).

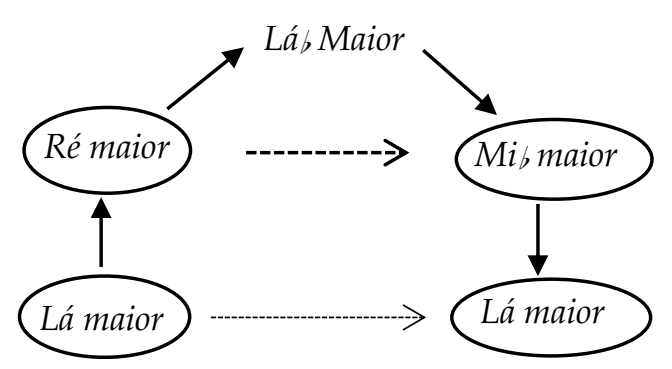

Figura 5: Nepomuceno, Artémis - Interlúdio, c. 626-723; síntese cadencial dentro do Diagrama de conexões proposto por Kopp 


\section{Conclusão}

Este trabalho esclarece como as propostas de Riemann e Kopp se fundem e se complementam, ao mesmo tempo em que são aplicáveis à peça de Nepomuceno, que claramente estrutura-se em tonalidade expandida.

Verificamos que analisar o texto de Nepomuceno por meio de uma ferramenta de análise harmônica ortodoxa se tornaria uma tarefa ímproba, senão inviável, visto que o compositor utiliza muitos acordes que não se inserem no contexto de campo harmônico tradicional.

Considerar que o repertório do final do século XIX e início do século XX representa uma extensão do sistema tonal é uma afirmação coerente, pois a análise constata que a estrutura cadencial da peça é visivelmente convencional, apresentando a organização mais recorrente possível: I, IV, V, I, onde o acorde de Mi, Maior é uma transformação cromática da dominante de Lá Maior.

O conceito de tonalidade expandida explica-se, portanto, pelo fato de se utilizar novos acordes, sonoridades e extensões, preservando a mesma estrutura cadencial que o sistema tonal estabeleceu desde sua sedimentação. Ao se nomear os acordes e realizar a análise com as relações do sistema de transformações cromáticas notamos que os intervalos formados entre os acordes são inerentes ao sistema tonal, quais sejam, uníssonos, terças, quintas e trítono. A diferença é que aparecem em uma nova roupagem, graças ao uso de cromatismo, o que corrobora a multiplicidade deste sistema, bem como a necessidade de um aprimoramento nas formas de analisá-lo.

Por meio da análise podemos ratificar o vanguardismo de Riemann, que esteve à frente de seu tempo enquanto teórico, bem como de Nepomuceno, que utilizou técnicas composicionais inovadoras como as transformações cromáticas e Kopp, que, por sua vez, deu à teoria de Riemann um formato adequado para aplicação em análises de peças deste período e com tal estrutura. Kopp estrutura sua ferramenta de análise em uma pesquisa extensa, explorando trabalhos de teóricos de vários períodos, atestando que essa preocupação constante inquietou e ocupou muitos estudiosos dentro da história da teoria musical. A despeito de sua ferramenta analítica simplificar o trabalho de investigação estrutural de repertório cromático, sua abrangência é muito mais profícua que a de outras propostas, que descartam uma grande parte do repertório por não tratarem da questão cromática. 
Desta forma, este artigo cumpre o propósito de divulgar essa ferramenta analítica, fomentando trabalhos analíticos que a apliquem, seja como forma de explorar e aprimorar essa ferramenta, seja como forma de conhecer a estrutura deste repertório sob uma nova perspectiva.

\section{Referências}

1. Kopp, David. 2002. Chromatic Transformations in Nineteenth-Century Music. New York: Cambridge University Press.

2. Rehding, Alexander. 2003. Hugo Riemann and the Birth os Modern Musical Thought. New York: Cambridge University Press.

3. Riemann, Hugo. 1893. Harmony Simplified: or, The Theory of the Tonal Fuctions of Chords. Londres: Augener Ltd.

4. 1929. Composición Musical. Barcelona: Labor.

5 . 1930. Armonia e Modulación. Barcelona: Labor.

6. . 2005. Teoria General de La Musica. Barcelona: Idea Books. 